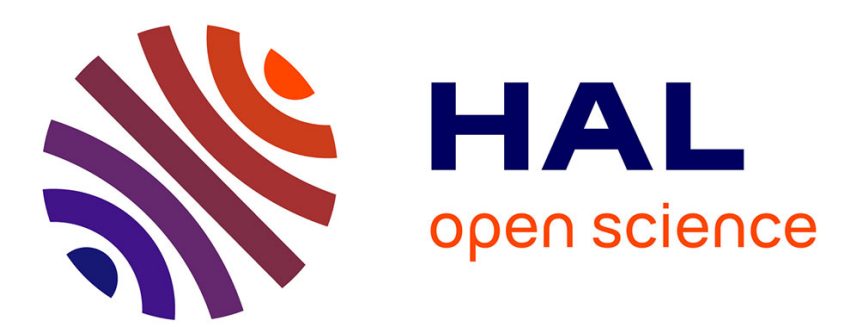

\title{
Continuum et maintien du lien social professionnel en situation de conflit verbal écrit : être poli ou impoli, mais y mettre les formes
}

Christina Romain, Béatrice Fracchiolla

\section{- To cite this version:}

Christina Romain, Béatrice Fracchiolla. Continuum et maintien du lien social professionnel en situation de conflit verbal écrit: être poli ou impoli, mais y mettre les formes. Langage et Société, 2021, Charles Goodwin: l'interaction au carrefour du langage, du corps et de la société, 173, pp.203-225. 10.3917/ls.173.0205 . hal-03088131v2

\section{HAL Id: hal-03088131 \\ https://hal.science/hal-03088131v2}

Submitted on 14 Dec 2021

HAL is a multi-disciplinary open access archive for the deposit and dissemination of scientific research documents, whether they are published or not. The documents may come from teaching and research institutions in France or abroad, or from public or private research centers.
L'archive ouverte pluridisciplinaire HAL, est destinée au dépôt et à la diffusion de documents scientifiques de niveau recherche, publiés ou non, émanant des établissements d'enseignement et de recherche français ou étrangers, des laboratoires publics ou privés. 


\author{
Béatrice Fracchiolla \\ Université de Lorraine \\ Centre de Recherche sur les Médiations EA3476 \\ En délégation au LEGS UMR 8238 \\ Beatrice.fracchiolla@univ-lorraine.fr

\section{Christina Romain} \\ Aix-Marseille Université - I.N.S.P.E. Aix-en-Provence \\ Laboratoire Parole et Langage UMR7309 CNRS \\ Christina.romain@univ-amu.fr
}

\title{
Titre : Continuum et maintien du lien social professionnel en situation de conflit verbal écrit : être poli ou impoli, mais y mettre les formes
}

\section{Résumé}

Le contexte même des échanges électroniques (interaction verbale asynchrone) les rend plus propices à la tension verbale : certains éléments (linguistiques et discursifs) y sont exacerbés en raison de l'absence d'autres éléments (prosodiques et mimogestuels) généralement présents dans les communications synchrones. En quoi et comment les échanges de courriels à plusieurs personnes en contexte institutionnel favorisent alors la cristallisation de la tension verbale et quels peuvent être leurs impacts sur le devenir de l'interaction verbale asynchrone ? Constitué de courriels de type CC échangés entre universitaires, notre corpus est étudié sous l'angle de l'analyse linguistique de la violence verbale (Fracchiolla et al., 2013a), en tant que montée en tension contextualisée se déclinant à travers différentes étapes dont: des actes de langage dépréciatifs, des marqueurs discursifs de rupture, des déclencheurs de conflits, et l'analyse du fonctionnement de l'agression verbale, de son impact et de sa gestion interactionnelle globale.

Mots clefs : politesse linguistique, courriels, milieu professionnel (universitaire), montée en tension verbale, faces.

Keywords linguistic politeness, emails, professional (university) environment, rise in verbal tension, faces.

Alors que la politesse contribue à la coopération interactionnelle et à la pérennité de la relation interdiscursive (Howard 1990), l'impolitesse est constitutive de tensions, de conflits interactionnels, et elle rend compte d'une menace à la face (Goffman 1973). Pourtant, tout échange ne repose pas sur la politesse (Watts 2003, Bousfield 2008, Culpeper 2008, Fracchiolla et Romain 2015, 2016). Notre cadre théorique repose sur la théorie des actes de langage (Austin 1962, Searle 1969) et les travaux sur la politesse (Lakoff 1973, Brown et Levinson 1987 ; Kerbrat-Orecchioni 2005), mais s'en distingue pour rejoindre le courant des recherches anglosaxonnes des années 2000 qui reposent, elles, sur une analyse de la politesse et de l'impolitesse au sein d'un même continuum (Eelen 2001 ; Watts 2003 ; Mills 2009 ; Locher et Watts 2005 ; Locher 2006 ; Bousfield 2008 ; Bousfield et Locher 2008 ; Archer 2017) et convoquent le contexte et l'analyse discursive, y compris dans une dimension pragmatique (Culpeper et al. 2003 ; Culpeper 2008 ; Bousfield 2008). Reprenant cette notion de continuum, nous l'illustrons dans le contexte unique de l'interaction verbale asynchrone (courriels) en milieu professionnel à travers l'étude de trois interactions montrant des nuances manifestes entre par exemple «politesse négative », «impolitesse positive» et «impolitesse négative ». Bien que déjà 
définies par Culpeper et al. (2003) et Brown et Levinson (1987), elles sont ici novatrices car situées dans la perspective d'un continuum qui tient compte du contexte, de la localisation du comportement verbal ; de plus, la notion de continuum y est vue dans la perspective discursive relationnelle de l'interaction verbale et de ses enjeux (Fracchiolla, 2013b). Nous questionnerons la place et la gestion de la tension verbale en répondant à ces questions :

i) en quoi la politesse permet-elle de favoriser la coopération interactionnelle écrite ?

ii) l'impolitesse conduit-elle toujours à la rupture interactionnelle?

iii) Ou peut-on la voir, via certains marqueurs linguistiques, comme caractéristique d'une dynamique relationnelle?

Depuis cinq ans, nos travaux (Fracchiolla et Romain 2014, 2015 ; Romain et Fracchiolla 2016) partent d'un constat : les courriels verbalement violents entre collègues au sein d'institutions universitaires, en particulier dans les courriels adressés en CC, sont en augmentation. Ils reposent sur un « effet tribunal» du CC combiné au déplacement progressif du conflit sur l'objet vers la personne ; ce conflit de personnes pré-existe souvent à l'échange de courriels, qui le réactive. Ces échanges constituent un terreau favorable à la (ré)émergence de la tension verbale : écrite, l'interaction laisse une trace et isole explicitement l'élément de discorde, ce qui a pour effet de lui assigner une place plus visible que dans une interaction orale.

Constitué de trois exemples de courriels échangés en CC entre universitaires dans le cadre de leurs activités professionnelles, le corpus porte ici sur l'expression écrite et la gestion interactionnelle de la menace. L'un montre une volonté d'accommodation; un autre, un refus de coopérer ; le dernier part d'un malentendu qui dégénère. Le corollaire à notre hypothèse de départ est que l'impolitesse comme la menace, ne conduisent pas per se à un échec de la communication : ne pas être d'accord ne signifie pas forcément ne pas vouloir coopérer. Or, ne pas être d'accord apparaît parfois comme une provocation, notamment au sein d'une équipe enseignante dont les relations interdiscursives sont sujettes à de fréquentes tensions. Le corpus illustrera ainsi une compréhension de la politesse et de l'impolitesse plus en nuances de dégradés que comme dichotomiques, pour révéler des enjeux parfois communs - dont la notion d'accommodation est une clef - au sens où "accommodation" renvoie au processus de (ré)ajustement à l'autre dans un échange potentiellement tendu (Giles et al. 1991).

\section{Le rapport de la politesse et de l'impolitesse aux différents types de cadres, synchrones ou asynchrones}

\subsection{Typologie des formes prises par la montée en tension}

En face à face, la montée en tension verbale connait diverses formes: montée en tension fulgurante (en diverses étapes marquées par des déclencheurs de conflit; des marqueurs discursifs de rupture et des actes de langage dépréciatifs directs à visée de domination); montée en tension polémique (montée en tension reposant sur des actes de langage indirects et implicites ; une argumentation et des figures de rhétorique à visée polémique et persuasive) ; montée en tension détournée - qui s'actualise dans des interactions feintes et ambiguës à valeur illocutoire contraire et enchâssées pour manipuler et harceler (Moïse et al. 2008 ; Fracchiolla et al. 2013). Ces études montrent comment des formes verbales attisent la tension, quand d'autres l'apaisent ; les descripteurs de la montée en tension en face à face sont identiques à ceux des échanges écrits asynchrones, qui ne bénéficient pas eux des divers éléments d'ajustement des échanges oraux (Romain et Fracchiolla 2016). Dans une interaction verbale en présence, l'adaptation directe à autrui permet la résorption de points de cristallisation du 
discours en tension - au contraire du courrier électronique, où les tensions sont difficiles à résoudre, ce qui favorise les montées en tension verbale. Pourtant, la politesse linguistique permet une régulation pour contrôler certains dérapages. Dans beaucoup d'interactions, elle est décrite comme la clé du lien social (Guéguen, 2008) promotrice de coopération, d'apaisement de la tension (Howard 1990), et contribuant à la pérennité de la relation interdiscursive.

\subsection{Les différents types de politesse}

Aussi, en contexte de tension potentielle, la politesse devient un cadre interdiscursif de ménagement de la face. Devant la menace que fait peser toute interaction sur les faces des interactants et dans la continuité des travaux de Goffman (1973), les mécanismes compensatoires sont décrits à travers la politesse positive et la politesse négative (Brown et Levinson 1987) qui visent chacune le ménagement d'une face. L'acception de Leech (1983) qui met plus au centre la personne à qui l'on s'adresse a notre préférence. La politesse positive tend de fait à produire du contenu discursif anti-menaçant : s'il vous plait ; bonne journée... là où la politesse négative atténue ou on évite les atteintes et les menaces aux faces à l'aide de procédés discursifs relevant de la modalisation du discours, l'atténuation et l'indirection : il faudrait; il semble que; j'aurai voulu savoir si, etc. Les formes qui s'y rapportent relèvent plus de l'ordre d'un choix individuel du locuteur que de formes vraiment ritualisées. Elle est un phénomène adaptatif qui fonctionne avec le degré de connaissance que l'on a de son interlocuteur et de la langue.

\subsection{Les différents types d'impolitesse}

Bousfield (2008) et Brown et Levinson (1987) mettent au centre de leurs analyses la préservation de la face (politesse); Culpeper y met, lui, l'attaque à la face et, par-là, le phénomène d'impolitesse (1996, 2005). Malgré cette différence, tous se sont d'abord accordés sur l'intentionnalité, définitoire. Culpeper (2010) s'en est ensuite désolidarisé car certains énoncés sont vus comme impolis, en contexte et par le destinataire, alors que ce dernier sait que son interactant ne voulait pas l'être. En ce sens Culpeper et al. (2003) et Bousfield (2008) distinguent l'impolitesse volontaire (selon un objectif visé) de l'involontaire (sans objectif visé ou contraire à celui qui est perçu du destinataire). Nous intéressant ici à l'émergence et au traitement interactionnel d'un différend observable dans des échanges de courriels pour y étudier ce qui crée de la tension verbale, le concept d'intention n'est pas questionné : la tension verbale qui émane des échanges vient selon nous de la réactivité du destinataire, pour qui est « impoli » le message qui lui est adressé (Hutchby 2008). Malgré toutes les distinctions définitoires opérées par Culpeper ${ }^{1}$ : nous considérons dans cet article comme complémentaires - et non systématiquement opposables - les deux faces, positive et négative, décrites par Goffman (1973) en termes de dynamique relationnelle entre les interactants (Arundale 2006 et Penman 1990). Bousfield distingue simplement l'impolitesse directe (vue comme menace directe) : «Ce ne fut qu'une réponse à la hauteur de la perversité de ton message et de son mensonge » (exemple 3$)^{2}$; de l'impolitesse indirecte (vue comme menace ou attaque indirecte d'autrui via l'implicature) : « Nous ne sommes pas une agence de tourisme! » (exemple 1) où l'inférence est qu'on ne peut accepter toutes les propositions de communication à un colloque.

\footnotetext{
${ }^{1}$ L'impolitesse directe (attaque de la face positive ou négative) ; l'impolitesse positive (attaque à la face positive) ; l'impolitesse négative (attaque à la face négative); le sarcasme et la politesse non sincère (attaque et ménagement non sincère); la suspension de la politesse (silence $v s$ stratégies de politesse)

${ }^{2}$ Les exemples sont extraits du corpus.
} 
Pour Watts (2003), qui considère l'existence d'interactions verbales neutres, sans marquage linguistique spécifique de type poli ou impoli, utiliser des marques de politesse ritualisées, socialement attendues a minima (Bonjour, Cordialement en français) n'est pas montrer de la politesse à valeur de ménagement de la face. Comme nous le verrons, cela se note particulièrement dans l'exemple 3, qui est d'une grande violence, bien qu'il contienne, justement, toutes les « marques rituelles » de la politesse. Nous pensons néanmoins que, dans les exemples étudiés, ces emplois ritualisés et conventionnels sont bien une marque volontaire de contrôle de la tension, en même temps qu'ils permettent à l'énonciateur de ménager sa propre face : on ne peut pas reprocher à une personne d'être impolie, si elle utilise des éléments de politesse conventionnelle a priori visibles et reconnaissables par tous comme tels (Fracchiolla 2011). Pour Terkourafi (2007), c'est le contexte, poli ou impoli, qui conditionne le caractère potentiellement impoli d'un mot ou d'une expression.

\section{L'accommodation}

La théorie de l'accommodation prend son origine dans le contexte multilingue des études sociolinguistiques (Giles et al. 1991). Elle explique le fonctionnement du recours à la langue selon les motivations de l'individu, la relation interpersonnelle, l'appartenance ou non au groupe, etc. L'accommodation communicative s'oppose à la divergence communicative. En contexte de divergence la conscientisation du choix est plus importante qu'en contexte de convergence (Bourhis 1983). Si notre corpus repose sur une communication unilingue, l'accommodation interpersonnelle nous intéresse pourtant, car elle rend compte d'options relationnelles, de stratégies, choisies ou non par les interactants. En contexte de tension verbale, l'absence ou la présence de processus d'accommodation montre un tissage relationnel plus ou moins coopératif - terme que nous entendons ici comme une forme de bienveillance envers son interlocuteur, mais surtout, comme l'acceptation d'un fonctionnement interactionnel harmonieux, fluide (participation et inférences) - qui correspond au principe de politesse au sens de Leech (1983). Dans ce sens, l'accommodation a des implications pragmatiques sur la relation (Giles et al. 1991).

\section{Présentation et analyse du corpus}

\subsection{Présentation du corpus et méthodologie}

Nous avons certes observé que les échanges de courriels collectifs tendent à focaliser et faire émerger les divergences, mais ce sont l'absence de politesse linguistique et une utilisation polémique de l'impolitesse linguistique qui favorisent la réalisation de la montée en tension verbale écrite. La politesse linguistique s'avèrerait d'autant plus nécessaire en l'absence de face à face des interactants, qu'il n'y aurait pas d'autres moyens que ceux de l'écrit pour réguler l'interaction. Ainsi, le fait de ne pas utiliser des éléments de politesse conventionnelle peut entraîner des réponses qui montrent une mauvaise interprétation des propos, voire qui se font menaçantes face à ce qui est pris pour une agression (Fracchiolla 2013). C'est ici que l'absence de politesse conventionnelle, bien que dénuée d'intention menaçante à la face sur le plan illocutoire, peut parfois contribuer à la montée en tension interpersonnelle de différentes manières. De fait, certains actes de langage présentent une menace pour la face d'autrui alors même que l'intention de menacer n'est pas initialement présente (Archer 2008 ; Bousfield 2008 ; Culpeper 1996; Harris 2011, Grainger et Mills 2016). Nous allons désormais nous intéresser, à travers l'étude des courriels échangés dans trois contextes distincts, aux outils discursifs qui permettent de ménager et de maintenir la relation interdiscursive, comme de la 
menacer, dans la mesure où nous envisageons politesse et impolitesse comme appartenant à un même continuum.

\subsection{Analyse du corpus}

Le corpus et ses résultats reposent sur une analyse de données empiriques, recueillies entre 2010 et 2012 grâce à un appel adressé aux collègues de toutes disciplines sur diverses listes de diffusion, au moyen d'une adresse spécifique créée sur yahoo.com. Nous avons ainsi obtenu un total de douze échanges thématiques en milieu universitaire (transmis par douze collègues issus de différentes universités françaises) ; tous avaient pour caractéristiques d'avoir été échangés en $\mathrm{CC}$, de rendre compte d'une tension verbale entre seulement deux interactants et d'être constitués de trois à six courriels. Une fois le corpus constitué, nous avons anonymisé le contenu des courriels afin de supprimer tout élément qui aurait pu permettre d'identifier les interactants et leur lieu d'exercice professionnel. Nous avons sélectionné trois échanges de ce corpus, dont le déroulement nous permet d'expliquer les trois formes de tension verbale écrite dont nous souhaitons rendre compte ici. Ces trois échanges correspondent donc aux trois exemples que nous avons choisis d'analyser ci-après. Pour chacun de ces exemples, nous faisons une analyse discursive de la montée en tension verbale orientée sur la place de la politesse et de l'impolitesse linguistique ; l'analyse graphique est ici mise de côté au profit de la dimension discursive et relationnelle des échanges.

\section{Exemple 1}

L'exemple 1 est constitué de trois courriels et met en scène trois collègues. Son objet concerne une difficulté sur l'horaire de passage pour une communication orale à un colloque. Alors que trois interventions sont prévues, seules deux sont possibles dans les temps impartis. Une négociation s'initie sur les possibilités de satisfaire tout le monde et pour trouver une solution. Dans cet exemple, la question des places de chaque personne est aussi en jeu : les interventions sont financées par les laboratoires, il y a donc une question de reconnaissance professionnelle et institutionnelle, liée à la face. Comment choisir en effet quelle personne sur les trois devrait renoncer à sa participation sans susciter un conflit ? Proposition est faite de réduire les temps de passage, ce qui signifierait néanmoins une inégalité de traitement par rapport aux autres interventions. Après consultation des collègues concerné·es, la personne à l'origine du courriel décide (et promet) finalement de trouver une solution sans léser personne.

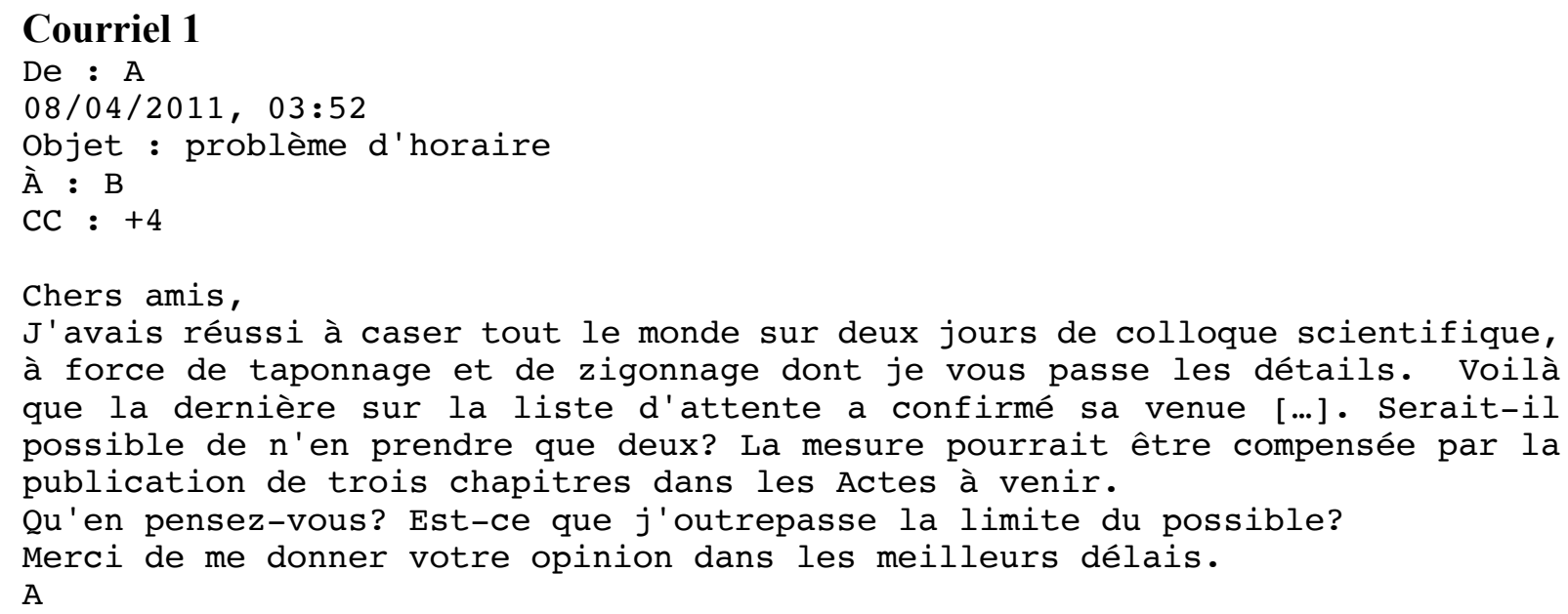


Ce premier courriel expose un problème d'organisation. Il commence sur une formule de politesse intentionnelle et sociale « Chers amis » avec une adresse de type affectif, qui crée du lien ; en termes d'acte de langage, cela correspond à de la politesse positive sur le plan illocutoire. Celle-ci est suivie d'une modalisation énonciative par l'imparfait « $\mathrm{j}$ 'avais réussi à », laissant présager que la situation est modifiée au moment de l'énonciation. Cette modalisation s'accompagne d'une formule curieuse et elliptique dans laquelle « taponnage » et «zigonnage $»^{3}$ cumulés renvoient aux efforts fournis pour contenter tout le monde ; mais ces efforts sont un échec en raison d'un événement extérieur. La responsabilité de l'échec est dépersonnalisée et reportée sur cet événement au moyen de la locution «voilà que » qui l'introduit - avec la dimension rhétorique du coup de théâtre. La modalisation se perpétue dans l'enchaînement de deux conditionnels et les trois questions directes posées - qui sont ici des précautions oratoires, et correspondent à un ménagement de la face - via une politesse négative. La présence de politesse négative (à travers les questions et l'argumentation menaçante, mais précautionneuse « serait-il possible de $n$ 'en prendre que deux ? ») permet dans cette situation de ménager les faces. Parallèlement, on trouve également de l'impolitesse positive dans la mesure où $A$ recherche une compensation, une négociation au sens courant du perdant/donnant. L'argumentation de A offre une publication "compensatoire" dans les actes, en contrepartie de ne pas pouvoir communiquer (recherche d'accommodation, d'alignement de positionnement); alors que le fait de communiquer va avec le fait de pouvoir publier. La sanction de ne pas pouvoir communiquer demeure donc inchangée. Dans cette mesure, il s'agit bien d'impolitesse positive : on présente quelque chose comme - + alors qu'il s'agit en fait toujours de -. La négociation reste déséquilibrée. La compensation n'en est pas vraiment une dans la mesure où tous les communicants auront droit à une publication dans les actes. C'est donc l'absence de possibilité de présenter à l'oral qui subsiste.

\section{Courriel 2}

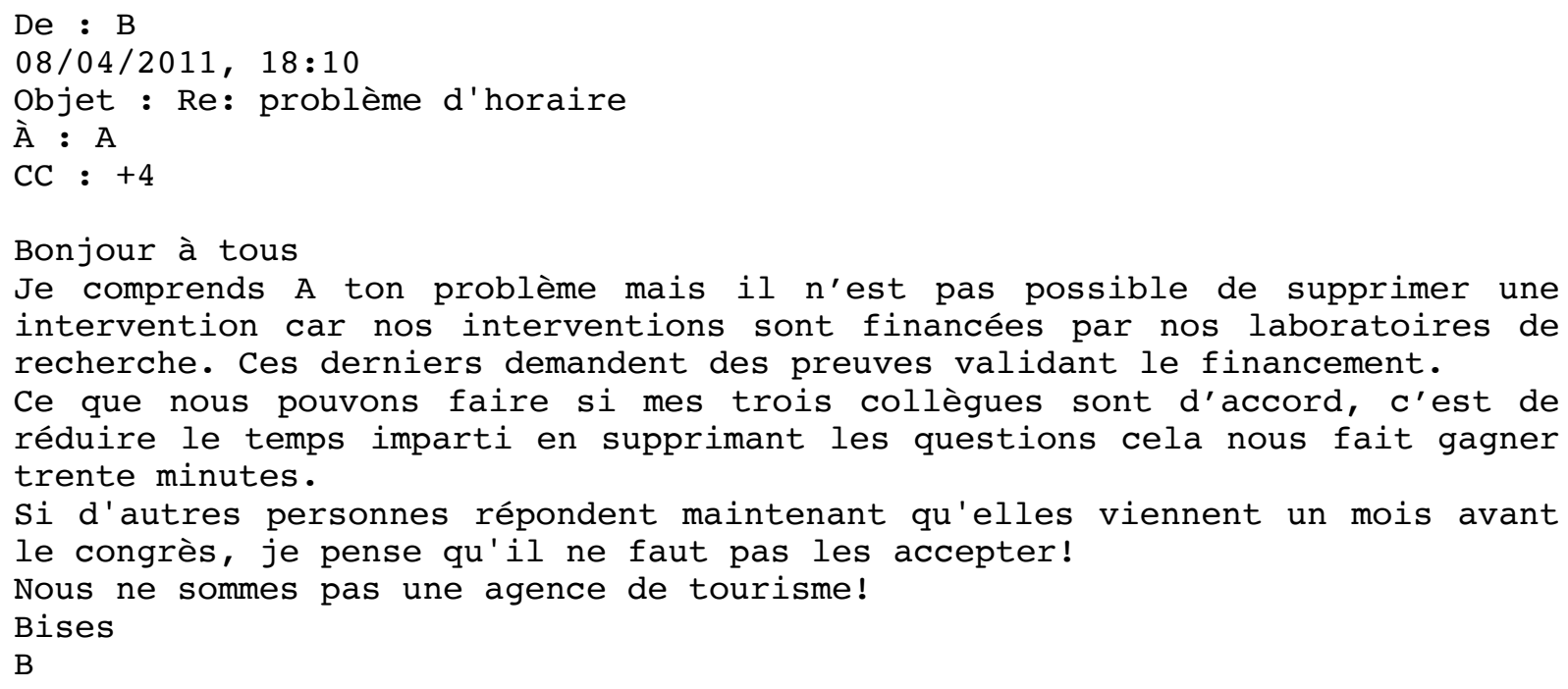

Le courriel commence par « Bonjour à tous », soit de la politesse positive. À cela succède une formule adoucissante visant l'accommodation «Je comprends A ton problème», immédiatement suivie de la formule «mais il n'est pas possible de supprimer une

\footnotetext{
${ }^{3}$ L'énonciatrice est canadienne. Pour «zigonnage », la définition du Petit Robert est « faire des essais en divers sens sans savoir s'y prendre »; «tenter de se frayer un passage en zigzaguant». Pour « taponnage », c'est un substantif construit sur le verbe « taponner » (perdre son temps, tatonner), aussi employé en français du Canada.
} 
intervention " ; c'est une réponse directe à ce qui a été pris pour une menace directe à la face (professionnelle, où ne pas participer signifie ne pas avoir son nom sur le programme, et donc n'être ni financé ni publié). La formule adoucissante visant l'accommodation est directement articulée à un refus "il n'est pas possible» ce qui s'interprète en politesse négative. L'argumentation non polémique qui suit vaut explication du refus : « car nos interventions sont financées par nos laboratoires de recherche. Ces derniers demandent des preuves validant le financement $»$. Ce type d'argumentation discursive non polémique est constitutive de ce que nous appelons désormais impolitesse positive.

Cette impolitesse positive se poursuit dans le courriel « Ce que nous pouvons faire si mes trois collègues sont d'accord, c'est de réduire le temps imparti en supprimant les questions ». On est contre, « mais », on essaie de trouver des solutions et de les promouvoir (« cela nous fait gagner trente minutes. ») : la négociation est à l'œuvre.

La phrase suivante est une solution intermédiaire en trois temps, avec deux niveaux de modalisation qui précèdent la proposition résolutive à proprement parler $1 /$ « ce que nous pouvons faire », 2/《 si mes trois collègues sont d'accord » (accommodation), " c'est de... » suivi 3/par la conclusion introduite par les deux points. A affirme donc sa volonté de coopérer.

Le courriel finit sur un focus pour recentrer le discours sur l'objet à l'origine du problème : l'élément perturbateur ne doit pas prendre le pas sur les conférenciers qui sont engagés depuis le début. Ici, la « personne » qui a surgi et perturbé le programme est perçue comme menaçante. C'est donc sur la réfutation d'accommoder cette personne, plutôt que celles engagées de longue date, que porte la dernière réflexion exclamative qui vaut pour de l'impolitesse négative : « $\mathrm{Si}$ d'autres personnes répondent qu'elles viennent un mois avant le congrès, je pense qu'il ne faut pas les accepter! ». La suite : « Nous ne sommes pas une agence de tourisme! » reste dans l'impolitesse négative, mais avec une dimension implicite : il s'agit d'une double attaque polémique indirecte reposant notamment sur l'ironie ; à la fois en direction de A qui cherche à contenter tout le monde ; et aussi en direction de la personne absente de l'échange, mais qui menace la relation entre $\mathrm{A}$ et $\mathrm{B}$ et qui est à l'origine de tout l'échange.

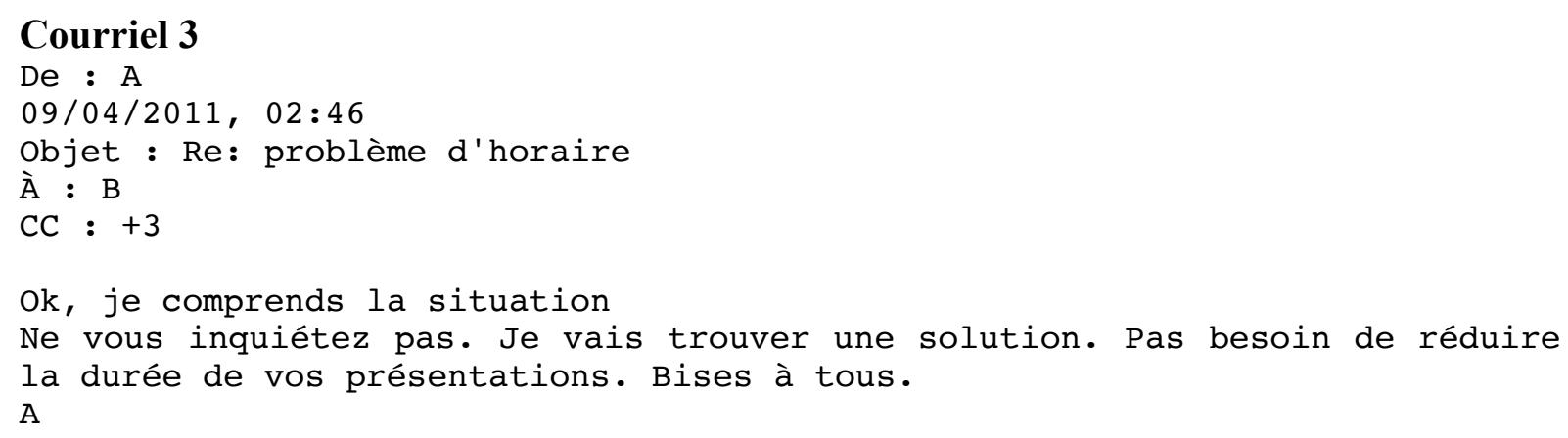

Entièrement fondé sur de la politesse positive (« ok », « ne vous inquiétez pas », « je comprends la situation », « je vais trouver une solution ») qui montre la réussite globale de l'interaction du point de vue de $\mathrm{B}$, ce dernier courriel conclusif montre que A souscrit à l'argumentation de B.

Cette première analyse met déjà certaines choses en évidence relativement à l'idée de continuum que nous développons ici. D'une part, nous avons la politesse positive, qui est constituée de « mots » isolés (« Bonjour », « Bises à tous », « Bonjour à tous », chères amies », «merci », etc). On trouve ensuite la politesse négative, qui est présente à travers des adoucisseurs ou atténuateurs tels que la modalisation avec le conditionnel ou des formules interrogatives directes. Ensuite, on trouve du discours, de l'argumentation mais qui ne va pas 
jusqu'à la polémique. Les arguments, qui cherchent à convaincre par la raison, menacent la face via le développement discursif sans être polémiques. C'est dans ce cadre que l'on parle ici d'impolitesse positive, au sens de favorable à la négociation, au fait de continuer à interagir ensemble. Cet exemple 1 montre une volonté intentionnelle de coopérer au sens de s'entendre et avancer ensemble, prouvée par l'argumentation non polémique associée à la politesse positive.

\section{Exemple 2}

Le deuxième exemple est constitué de trois courriels et met en scène une interaction directe entre deux collègues, avec un total de neuf collègues destinataires. La tension se focalise sur l'interprétation du mot " urgent » écrit en capitale dans le titre du courriel 1, reprise à la fin par «merci pour vos réponses rapides». À partir de cette indication, une personne répond de manière rapide, directe et négative à la question qui est posée. Une montée en tension s'ensuit.

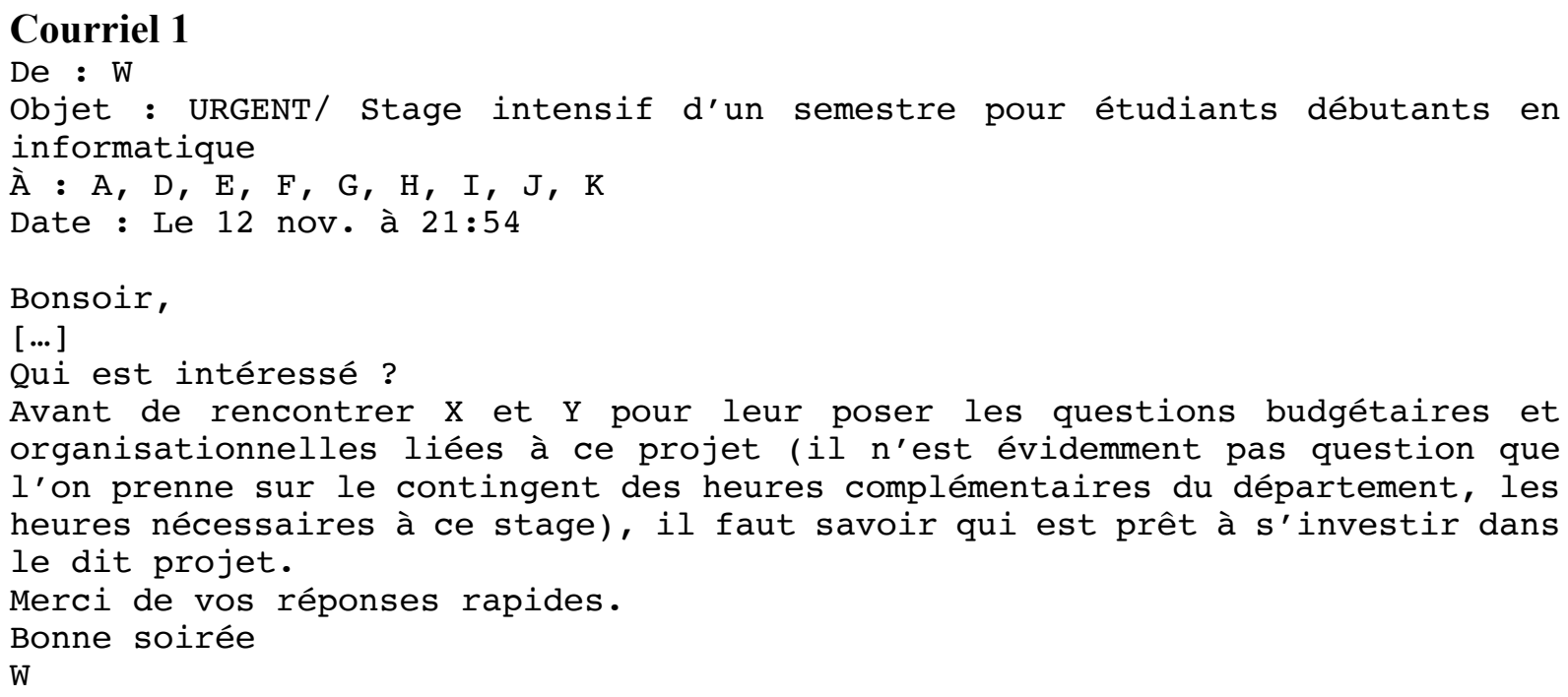

\section{Courriel 2}

\section{De : A}

Objet : Re : Urgent / Stage intensif d'un semestre pour étudiants débutants en informatique

Dans le courriel 1, W utilise des éléments minimaux de politesse positive : «Bonsoir » «Bonne soirée ", indiquant ainsi que le courriel est écrit hors horaires de bureau. Ce fait accentue l'impression d'urgence à répondre. Le lendemain matin, à 9h03, l'un des destinataires répond négativement à la demande en compensant par un adoucisseur qui rend compte d'une politesse négative ( «je suis désolé ») et en utilisant des éléments ritualisés de politesse positive ( Chers tous », « amitiés »). À cela s'articule une réfutation argumentée, à partir de « mais non »: «je 
ne suis pas disponible pour m'investir », qui bascule dans la polémique en raison de l'ajout généralement interprété comme axiologisant à nuance péjorative du syntagme "ce type » devant le mot «projet»; la phrase serait en effet restée de l'ordre de la seule réfutation argumentée si la réponse avait été « je ne suis pas disponible pour ce projet». Or, l'ajout de « ce type de projet » en permet une interprétation polémique, car l'expression semble discréditer le projet lui-même.

Cette polémicité est confirmée par l'ajout apposé de " pas dans ces conditions », qui cristallise la tension en créant de l'implicite («ces conditions » sont discutables). L'implicite laisse entendre qu'une possibilité d'acceptation aurait pu se réaliser mais dans d'autres conditions, que celles présentes.

Le refus appuyé sur des arguments polémiques de $\mathrm{A}$ fait entendre à $\mathrm{W}$ qu'il ne changera pas (facilement du moins) d'avis. Le lien relationnel est entamé discursivement.

Le contenu interdiscursif, relationnel, peut s'interpréter aussi comme relevant d'une forme de maladresse (y a-t-il un implicite péjoratif visant la formulation adressée par $\mathrm{W}$ ou bien A rendil juste compte de l'urgence à répondre et réaliser le projet qu'il ne peut satisfaire ?). A manifeste bien un intérêt pour le projet, mais non dans les conditions de réalisation proposées, ce qui laisse à $\mathrm{W}$ deux choix d'interprétation :

- la dimension argumentative : A explique qu'il ne peut satisfaire à la demande et répond dans l'urgence.

- ou la version polémique : A remet en cause les conditions de la proposition de $\mathrm{W}$ et donc sa proposition elle-même. La réponse succincte et rapide dans l'écriture, en devient ramassée et donc implicite.

L'interprétation selon laquelle l'impolitesse (donc le versant le plus polémique) attise les émotions (Culpeper et al. 2014) a fortiori négatives, nous fait penser que $\mathrm{W}$ interprètera cette réponse en attaque; ce que confirme sa réponse.

\section{Courriel 3}

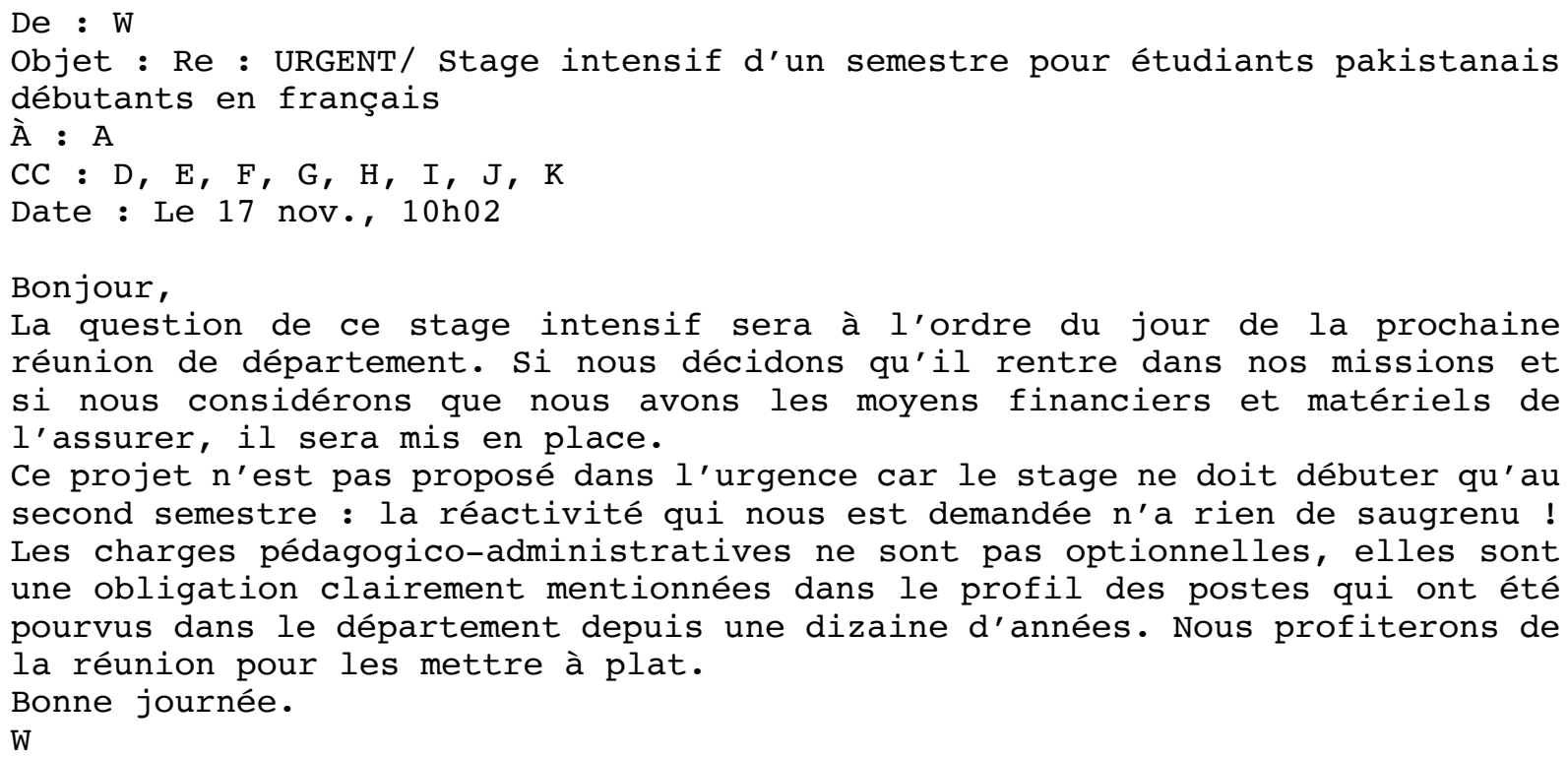

W utilise à nouveau dans ce dernier courriel de la politesse positive minimale : «Bonjour », «Bonne journée ». Néanmoins, le positionnement agonal de $\mathrm{W}$ est visible immédiatement après : ses choix argumentatifs montrent que $\mathrm{W}$ se sent menacé par la réponse de $\mathrm{A}$ : son deuxième courriel renvoie aux obligations de service et se transforme donc en menace envers les personnes qui ne rempliraient pas leurs tâches («Les charges pédagogico-administratives ne sont pas optionnelles, elles sont une obligation clairement mentionnées dans le profil des 
postes qui ont été pourvus dans le département depuis une dizaine d'années »). Ce rappel implicite et curieux (W a-t-il le sentiment que le refus d'une seule personne, A, pourrait remettre en cause le projet en lui-même ? que les autres feront pareil ?) s'accompagne de l'affirmation que la décision du stage sera prise en réunion de département - soit à une date ultérieure non définie qui ne rentre plus dans l'urgence. Ce courriel 3 offre un retournement de situation par rapport à ce qui est demandé au départ. Il n'y est plus question d'urgence - qui est niée, ni de volontariat, mais de « mission », de « mise en place », de « non optionnel », d' " obligation ». Cette situation rend compte de ce que nous avons appelé impolitesse négative : des menaces qui reposent sur des actes de langage polémiques sont formulées, provoquant le discrédit, la disqualification de A. De plus, l'attaque polémique de W est renforcée par l'utilisation du terme « saugrenu » qui est axiologiquement péjoratif.

\section{Exemple 3}

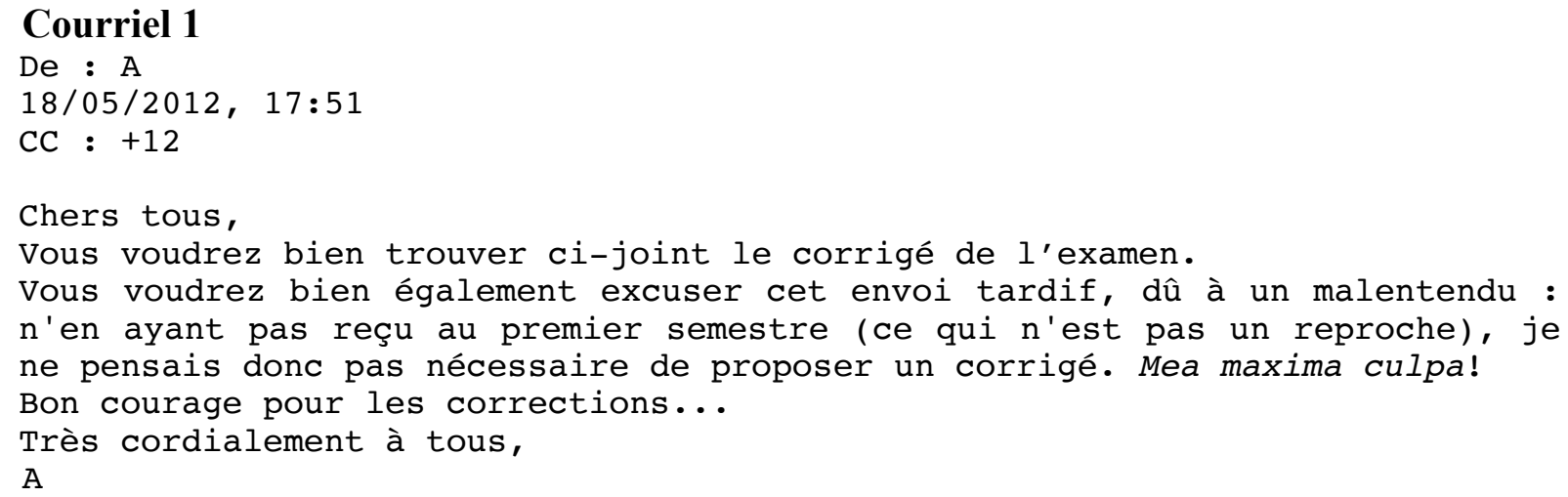

Dans cet ultime exemple, le collègue en charge du sujet d'examen du second semestre envoie un corrigé aux douze collègues co-correcteurs. Il explique qu'il le fait tardivement car il ne pensait pas devoir envoyer un corrigé n'en ayant pas lui-même reçu au premier semestre. Le courriel 1 montre un travail au niveau de la politesse positive de la part de A " Chers tous ", « très cordialement à tous », et il s'excuse par deux fois « excuser », " Mea maxima culpa! » pour cette omission et cet envoi tardif. B (qui était chargé du sujet/corrigé du premier semestre) lui répond alors :

\section{Courriel 2}

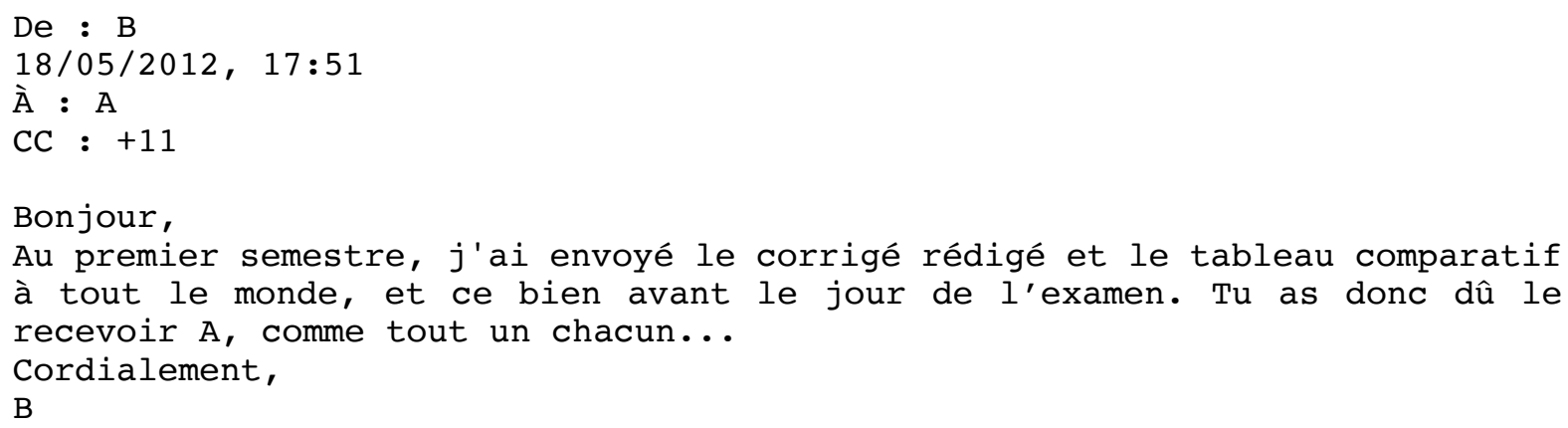

$\mathrm{B}$ répond à $\mathrm{A}$, les onze autres correcteurs en copie $\mathrm{CC}$; le courriel s'ouvre et se ferme par des formules rituelles de politesse positive typiques de ces échanges en milieu académique. La première phrase s'articule en deux segments : le premier reprend chronologiquement les éléments factuels ; le second s'articule en une attaque polémique indirecte à partir de la conjonction de coordination « et» (« et ce bien avant le jour de l'examen. Tu as donc dû le recevoir $\mathrm{A}$, comme tout un chacun... »). Contrairement à ce qu'il dit, A ne peut pas ne pas avoir 
reçu le corrigé. L'accommodation négative réside alors dans la négociation pour savoir a) qui de $\mathrm{B}$ ou de $\mathrm{A}$ a commis une erreur (ou « faute " professionnelle); ou b) lequel des deux ment : B (qui n'aurait pas envoyé le corrigé), ou A (qui l'aurait en fait reçu). En effet, tous les autres collègues l'ont reçu ; par conséquent, pour $\mathrm{B}$, A est de mauvaise foi ( $\mathrm{B}$ ne considère pas d'autres possibilités : le courriel électronique a pu se perdre ou ne pas être traité involontairement). Du point de vue illocutoire, ce courriel résulte en une disqualification de A par B via une utilisation d'impolitesse négative sous la forme d'une attaque polémique indirecte.

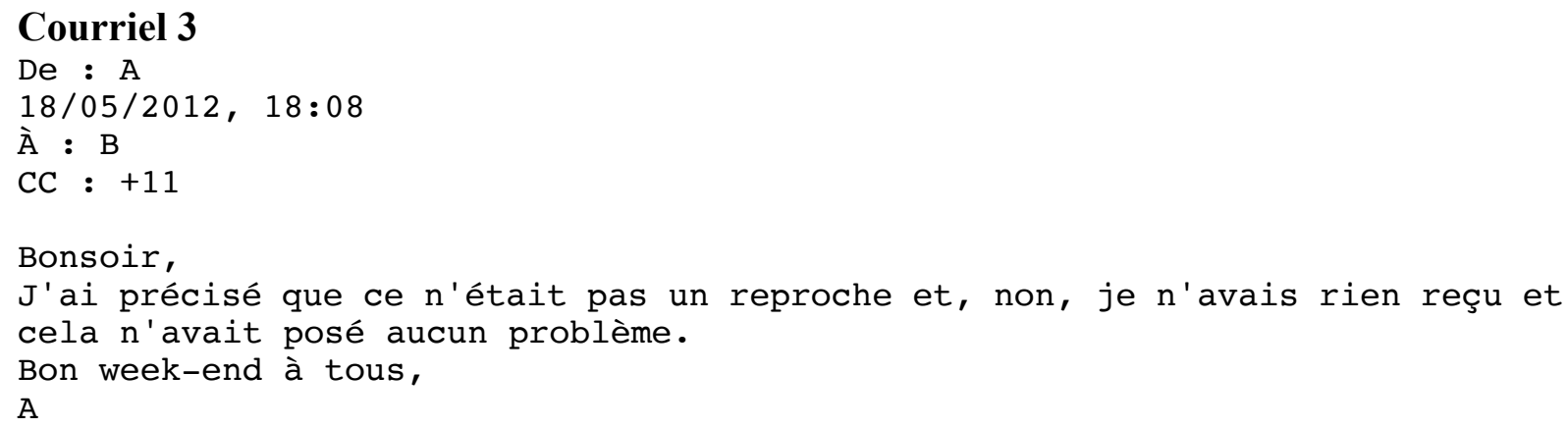

A répond aussi avec des éléments de politesse positive en ouverture « Bonsoir », qui sont encore plus clairement situés en clôture : nous sommes le vendredi soir («Bon week-end à tous »). A réfute l'attaque polémique qui le discrédite, précise que sa demande était dépourvue d'intention polémique envers $\mathrm{B}$ et cherche une issue positive à la tension ("J'ai précisé que ce n'était pas un reproche et, non, je n'avais rien reçu et cela n'avait posé aucun problème »). A cherche à apaiser qu'il interprète comme un point de cristallisation et de tension pour B ; A ménage ensuite la face de B : cela n'a pas posé de problème. Le cadre illocutoire est celui de la politesse négative, A fourni un travail interactionnel pour maintenir le lien social et désamorcer la tension.

\section{Courriel 4}

$\mathrm{De}: \mathrm{B}$

$19 / 03 / 2016,07: 51$

À : $\mathrm{A}$

$\mathrm{CC}:+11$

Bonjour,

Il est navrant que tu sois le seul à n'avoir pas reçu le message, à n'avoir pas demandé qui avait fait le corrigé As-tu eu le sujet au moins ?... mais cela ne doit pas être non plus un problème...

Bon week-end,

B

Le courriel s'ouvre et se ferme sur des éléments de politesse positive minimalistes (« Bonjour », «Bon week-end»- mais pas « à tous » par exemple, contrairement au précédent courriel de A). B enchaîne sur une attaque polémique directe à $A$, qui oriente tout le courriel : « il est navrant que tu » et continue par deux questions directes à sens implicite et ironique, laissées en suspens au niveau du sens dont l'objectif est délibérément de se moquer de A devant les autres en le disqualifiant : "As-tu eu le sujet au moins ?... mais cela ne doit pas être non plus un problème... ». Les attaques répétées de $\mathrm{B}$ vers $\mathrm{A}$ représentent ici des actes de violence verbale détournée (la formulation nécessite une interprétation) et polémique qui s'inscrivent dans le continuum en termes d'impolitesse négative, mais qui nous semble se trouver au point maximal de ce que l'on peut atteindre en termes d'attaques. Il s'agit ici d'un conflit de personnes, qui ne se situe plus sur le plan de la raison et de l'argumentation comme dans les deux premiers 
exemples analysés, mais sur le plan émotionnel. Bien que formulées de manière indirecte, les attaques portent ici sur la personne et ses compétences pour occuper la place professionnelle qu'elle occupe et non plus sur le « corrigé d'examen ». C'est dans cette mesure que le point limite entre impolitesse négative et violence verbale à l'égard de A est atteint.

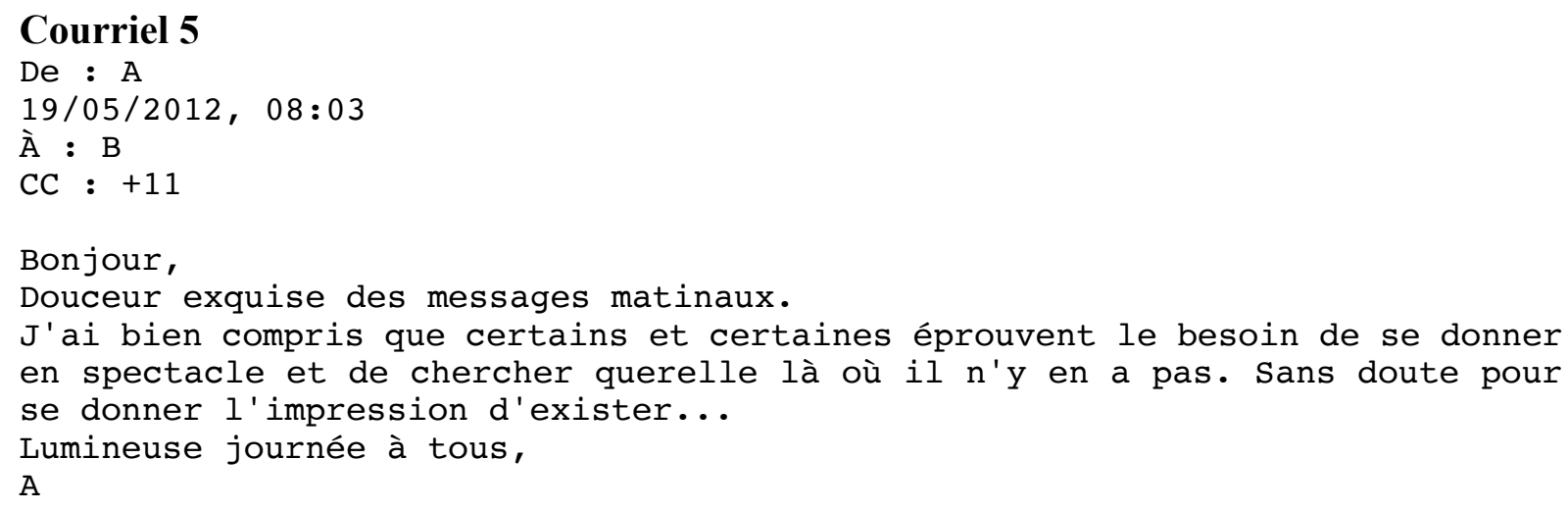

Dans le courriel 5, A utilise le cadre de la politesse positive en ouverture et en clôture, en l'accentuant néanmoins dans la clôture par une formule inhabituelle et créative (donc non rituelle), qui invite à se tourner vers la lumière $\mathrm{A}$ répond à l'attaque portée par $\mathrm{B}$. Néanmoins, A dépersonnalise son attaque en ne prenant pas $\mathrm{B}$ directement à partie : « J'ai bien compris que certains et certaines éprouvent le besoin de se donner en spectacle et de chercher querelle là où il n'y en a pas ». Le fait d'utiliser ici le double pronom « certains et certaines » au masculin et au féminin participe de cet effet de généralisation, dépersonnalisant. Suit une menace et attaque polémique indirecte à la face de B : "Sans doute pour se donner l'impression d'exister », qui constitue une exacerbation de la tension. Le cadre est toujours ici celui de l'impolitesse négative. A prend d'une certaine manière acte que $\mathrm{B}$ a déplacé le conflit sur la personne et répond donc sur cet axe également. Le conflit interpersonnel est déplacé sur la fiabilité professionnelle de l'un par rapport à l'autre.

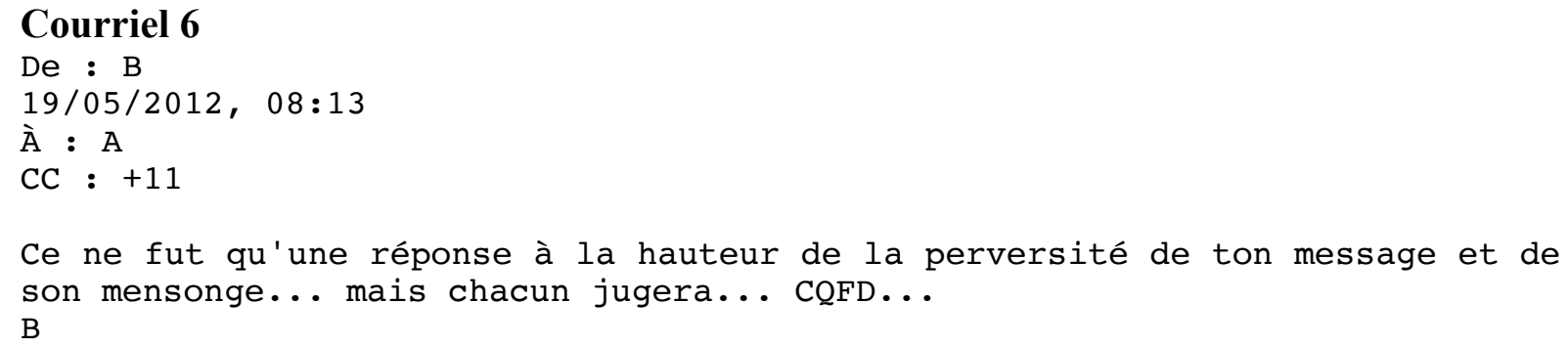

Dans cette réponse, on comprend clairement que B s'inscrit toujours dans la ligne de l'impolitesse négative en reprenant et poursuivant, les termes de l'attaque polémique initiée à l'égard de A «à la hauteur de la perversité de ton message et de son mensonge ». Néanmoins, tout en attaquant A, il prend soin de dépersonnaliser l'attaque autant qu'il le peut ; d'une part, ce n'est pas A « qui est accusé de mensonge », mais « son message »; d'autre part, à travers l'usage des pronoms et déterminant (indéfini) «ce ne fut qu'une réponse » (ce n'est donc pas « sa » réponse). Autrement dit, l'attaque personnelle est ici détournée à travers des « objets » qui deviennent les interactants à la place des personnes. Cet effet de dépersonnalisation et de rappel à l'objet du conflit (A a voulu le mettre en défaut) a pour effet de permettre à la tension qui menaçait d'aller vers de la violence verbale de retomber et de rester dans l'impolitesse négative, tout en s'en remettant au jugement de l'ensemble des collègues, qui sont ici interpellés « chacun jugera... ». 
L'impolitesse négative caractérise cet exemple 3 car toutes les attaques qui sont portées, le sont via des procédés indirects qu'il s'agisse d'ironie, d'utilisation de l'implicite (courriel 4) ou encore de dépersonnalisation des attaques (courriel 5 et 6). C'est finalement ce phénomène d'indirection constante qui permet de rester dans l'impolitesse négative, sans basculer dans de la violence verbale directe.

\section{Conclusion}

L'analyse nous conduit ainsi à distinguer l'impolitesse positive de l'impolitesse négative. L'impolitesse positive se définit comme une menace à la face qui se développe sur un registre argumentatif à propos de l'élément constitutif du point de cristallisation. Dans l'impolitesse positive, le contenu discursif et le développement argumentatif autour de l'objet discuté se situent sur le plan rationnel (de la raison) : la forme même prise par l'argumentation constitue en soi un type de ménagement inscrit dans la poursuite du lien relationnel. L'impolitesse positive correspond de fait à une recherche d'accommodation, d'alignement entre soi et autrui. Si l'échange, bien que positif, tend néanmoins vers l'impolitesse, cela est dû à la dimension persuasive - et en cela contraignante du point de vue illocutoire - de l'échange. L'impolitesse négative s'en distingue car elle déborde de l'objet à l'origine de la tension et se développe en une attaque polémique qui tend à créer, réactiver, voire démultiplier de nouveaux points de cristallisation à l'égard de la personne destinataire, via la tonalité polémique. Usant de la menace et de l'attaque (directe et indirecte), elle ne laisse à l'autre ou à ses idées aucune possibilité d'exister discursivement dans l'écrit lui-même ; et fait ainsi basculer l'échange dans le type d'impolitesse linguistique le plus éloigné sur l'échelle du continuum qui s'étend de la politesse à l'impolitesse. Nous confirmons ainsi l'hypothèse posée en introduction d'un continuum qui s'articule en quatre étapes, sans rupture : partant de la politesse positive (1), il se déploie en politesse négative (2), puis en impolitesse positive (3) pour arriver à l'extrême à l'impolitesse négative (4) - laquelle marque un point de bascule frontière vers la violence verbale, rarement franchi en raison du haut risque de perte de face qui lui est associé.

Notre étude met en évidence trois résultats par rapport à cette idée de continuum :

i) l'impolitesse n'est pas nécessairement négative pour l'interaction verbale : celle que nous nommons positive est motrice dans la progression, l'accommodation, la réalisation de l'échange entre les interactants. Ne pas être d'accord ne signifie pas ne pas vouloir coopérer dans l'échange : formuler un avis argumenté contraire en est un exemple. En revanche, ne pas être d'accord et ne pas vouloir coopérer est du domaine de l'attaque polémique (impolitesse négative) ; attaque qui peut être directe ou indirecte (comme l'ironie) (cf. notion de polirudesse, Kerbrat-Orecchioni 2005).

ii) Le principal marquage de la frontière entre impolitesse négative et violence verbale passe par l'indirection de l'attaque ; indirection qui, tout en prenant appui sur une variété importante d'éléments de langage liés aux mots, aux discours, est toujours fondée sur l'adresse et l'interpellation.

iii) Le troisième résultat porte enfin sur la coopération. Trois attitudes distinctes sont observables à l'aune des trois exemples analysés : une intention de coopérer existe (exemple 1) ; de ne pas coopérer de la part d'un ou de plusieurs interactants (exemple 3); ou bien encore une forme d'entre deux est générée sur le plan pragmatique par un malentendu, une mauvaise formulation de départ, une réponse trop rapide etc. (exemple 2). 
Le déroulé des échanges dans ces trois exemples met ainsi en évidence un continuum qui se situe sur deux plans différents, mais qui fonctionnent en parallèle : le premier plan est celui du continuum qui va de la politesse positive à l'impolitesse négative. Le second plan est celui qui suit le développement de chaque interaction autour des notions d'intentionnalité et de coopération. Or, on l'a vu, ces deux plans se rejoignent en certains points, en particulier lorsque l'intention est claire - mais pas lorsqu'elle fait l'objet d'un malentendu comme dans l'exemple 2. Dans tous les cas, le fait d'envoyer un message en CC implique une participation indirecte à l'interaction des autres récipiendaires, non scripteurs, de l'échange : ils en sont témoins, avec cette nuance près, qui pourrait faire l'objet d'un autre article encore, que lorsque l'intention est de ne pas coopérer, le courriel, envoyé en cc, devient alors une tribune.

\section{Références}

Archer D. (2017), « (Im)politeness in Legal Settings », dans Culpeper J., Haugh M. \& Kádár D.Z. (dir.), The Palgrave Handbook of Linguistic (Im)politeness, UK, Palgrave Macmillan, p. 713-737.

Archer D. (2008), "Verbal aggression and impoliteness: related or synonymous? », dans Bousfield D. \& Locher M. (dir.), Impoliteness in Language: Studies on its Interplay with Power in Theory and Practice, Berlin/New York, Mouton de Gruyter, p. 181-207.

Arundale R.B. (2006), «Face as relational and interactional: a communication framework for research on face, facework, and politeness », Journal of Politeness Research 2, p. 193-216.

Auger N., Fracchiolla B., Moïse C. \& Romain C. (2010), « Interpellation et violence verbale : essai de typologisation », Corela [En ligne] HS-8 | 2010, mis en ligne le 23 novembre 2010, consulté le 30 septembre 2016. URL : http://corela.revues.org/1023 ;DOI:10.4000/corela.1023 Austin J. L. (1962), How to do Things with Words, Oxford, Oxford University Press.

Bourhis R.Y. (1983), « Language attitudes and self-reports of French-English usage in Quebec ", Journal of Multilingual and Multicultural Development 4, p. 163-179.

Bousfield D. (2008), Impoliteness in Interaction, Amsterdam/Philadelphia, John Benjamins.

Bousfield D. \& Locher M. (dir.) (2008), Impoliteness in Language: Studies on its Interplay with Power in Theory and Practice, Berlin/New York, Mouton de Gruyter.

Brown P. \& Levinson S. (1987), Politeness. Some universals in language use, Cambridge, Cambridge University Press.

Buber M. (1992), Je et Tu, Paris, Aubier Montaigne.

Culpeper J. (2010), « Conventionalised impoliteness formulae », Journal of Pragmatics 42, p. 3232-3245.

Culpeper J. (2008), « Reflections on impoliteness, relational work and power », dans Bousfield D. \& Locher M. (dir.), Impoliteness in language, Berlin, Mouton de Gruyter, p. 17-44.

Culpeper J. (2005), « Impoliteness and entertainment in the television quiz show: The Weakest Link », Journal of Politeness Research 1, p. 35-72.

Culpeper J. (1996), « Towards an Anatomy of Impoliteness », Journal of Pragmatics 25, p. 349-367.

Culpeper J., Bousfield D. \& Wichman A. (2003), « Impoliteness revisited: with special reference to dynamic and prosodic aspects », Journal of pragmatics 35, p. 1545-1579.

Culpeper J., Schauer G. \& Marti L. (2014), « Impoliteness and emotions in a cross-cultural perspective », SPELL : Swiss papers in English language and literature 30, p.

67-88.

Eelen G. (2001), A Critique of Politeness Theories, Manchester, St. Jerome Publishing.

Fracchiolla B. (2011), « Politeness as a strategy of attack in a gendered political debate - The Royal-Sarkozy debate », Journal of Pragmatics 43(10), p. 2480-2488.

Fracchiolla B. (2013a), « De l'agression à la violence verbal », dans Fracchiolla B., Moïse 
C., Romain C. \& Auger N. (dir.), Violences verbales. Analyses, enjeux et perspectives, Rennes, Presses Universitaires de Rennes, p. 19-36.

Fracchiolla B. (2013b), Altérité énonciative et performativité des discours pour une linguistique relationnelle, Habilitation à diriger des recherches, Université Montpellier 3.

Fracchiolla B. (2017), « Performativité des constructions identitaires : Mariage pour tous, nom, adresse et filiation », Le discours et la langue. Revue de linguistique française et d'analyse de discours, Tome 9.2, p. ??- ??.

Fracchiolla B., Moïse C., Romain C. \& Auger N. (2013), «Introduction », dans Fracchiolla B., Moïse C., Romain C. \& Auger N. (dir.), Violences verbales. Analyses, enjeux et perspectives, Rennes, Presses Universitaires de Rennes, p. 9-16.

Fracchiolla B. \& Romain C. (2015), " Montée en tension et usage du courrier universitaire », dans Casanova R. \& Pesce S. (dir.), Violence en institutions : points de vue thématique, Rennes, Presses Universitaires de Rennes, p. 201-214.

Fracchiolla B. \& Romain C. (2014), « Réflexions pour une élaboration d'une charte éthique des relations interdiscursives : le cas de la messagerie universitaire », dans Colon de Carvajal I. \& Ollagnier-Beldame M. (dir.), Actes du colloque international Interactions Multimodales Par Ecran (IMPEC), p. 123-133. En ligne : http://impec.ens-lyon.fr/actes-du-colloque-impec2014-en-ligne-208378.kjsp

Giles H., Coupland J. \& Coupland N. (1991), Contexts of Accommodation. Developments in applied sociolinguistics, Cambridge, Cambridge University Press,

Goffman E. (1973), La mise en scène de la vie quotidienne, Paris, Editions de Minuit.

Grainger K. \& Mills S. (2016), "Language, Culture, (Im)politeness ans (in)directness », dans Grainger K. \& Mills S. (dir.), Directness and indirectness across cultures, UK, Palgrave Macmillan, p. 1-33.

Guéguen N. (2008), « La politesse, clé du lien social », Cerveau et Psycho 27, p. 28-31.

Harris S. (2011), « The limits of politeness re-visited: courtroom discourse as a case in point», dans Discursive Approaches to Politeness, Linguistic Politeness Research Group (dir.), Berlin/Boston, Mouton de Gruyter, p. 85-108.

Holtgraves T. (1992), « The linguistic realization of face management : implications for language production and comprehension, person perception, and cross-cultural communication ", Social Psychology Quarterly 552, p. 141-159.

Howard D. (1990), « The influence of verbal responses to common greetings on compliance behavior : The foot-in-the-mouth effect », Applied Social Psychology 20, p. 1185-1196.

Hutchby I. (2008), « Participants' orientations to interruptions, rudeness and other impolite acts in talk-in-interaction », Journal of Politeness Research 4(2), p. 221-241.

Kerbrat-Orecchioni C. (2005), Le discours en interaction, Paris, Armand Colin.

Lakoff R. (1973). « The Logic of Politeness ; or, Minding your p's and q's», dans The Ninth Conference on Performatives, Presupposition, and Implicatures, Arlington, Center for Applied Linguistics, p. 79-105.

Leech G. (1983), Principles of Pragmatics, London, Longman.

Locher M. A. (2006), « Polite behaviour within relational work. The discursive approach to politeness ", Multilingua 25(3), p. 249-267.

Locher M. A. \& Watts R. J. (2005), "Politeness theory and relational work », Journal of Politeness Research: Language, Behaviour, Culture 1(1), p. 9-33.

Mills S. (2009), "Impoliteness in a cultural context», Journal of Pragmatics 41, p. 1047-1060. Moïse C., Auger N., Fracchiolla B. \& Romain C. (dir.). (2008). La violence verbale.

Espaces politiques et médiatiques. Tome 1. Des perspectives historiques aux expériences éducatives. Tome 2, Paris, L'Harmattan.

Park H.S. (2008), « The effect of shared cognition on group satisfaction and performance : Politness and efficiency in group interaction », Communication Research 35(1), p. 88-108. 
Penman R. (1990), « Facework and politeness : multiple goals in courtroom discourse », Journal of Language and Social Psychology 9 (1-2), p. 15-39.

Romain C. \& Fracchiolla B. (2020), « Principe de coopération interactionnelle et agressivité. Corrélations avec les formes de politesse et d'impolitesse linguistiques en présence de conflit verbal dans la rédaction de courriels en milieu professionnel francophone », in CORELA, vol. 18-2, décembre 2020.

Romain C. \& Fracchiolla B. (2016), «Violence verbale et communication numérique écrite : la communication désincarnée en question », Cahiers de praxématique 66 |2016. En ligne : http://praxematique.revues.org/4263

Searle J. R. (1969), Speech Acts, Cambridge, Cambridge University Press.Terkourafi M. (2007), From Politeness to Impoliteness: the Framed-Based Approach, Cambridge, Cambridge University Press.

Watts R. (2003), Politeness, Cambridge, Cambridge University Press. 